\title{
Papers
}

\section{Palliative chemotherapy for advanced colorectal cancer: systematic review and meta-analysis}

\author{
Colorectal Cancer Collaborative Group
}

\begin{abstract}
Objectives To determine the benefits and harms of palliative chemotherapy in patients with locally advanced or metastatic colorectal cancer and to compare the outcomes for elderly and younger patients.

Design Meta-analysis of individual patient data and published summary statistics from trials for which individual patient data could not be obtained from the investigators.

Studies All randomised controlled trials comparing palliative chemotherapy with supportive care in patients with advanced colorectal cancer that were identified by computerised and hand searches of the literature, scanning references, and contacting investigators.

Main outcome measures Survival, disease progression, quality of life, and toxicity.

Results 13 randomised controlled trials including a total of 1365 patients met the inclusion criteria. Meta-analysis of seven trials that provided individual patient data (866 patients) showed that palliative chemotherapy was associated with a $35 \%$ reduction in the risk of death (95\% confidence interval $24 \%$ to $44 \%)$. This translates into an absolute improvement in survival of $16 \%$ at both six and 12 months and an improvement in median survival of 3.7 months. No age related differences were found in the effectiveness of chemotherapy, but elderly patients were under represented in trials. The overall quality of evidence relating to treatment toxicity, symptom control, and quality of life was poor.

Conclusions Chemotherapy is effective in prolonging time to disease progression and survival in patients with advanced colorectal cancer. The survival benefit may be underestimated in this analysis as some patients in the control arms received chemotherapy.
\end{abstract}

\section{Introduction}

Colorectal cancer causes 15000 deaths each year in England and Wales and is the second most common cause of cancer death. ${ }^{12}$ The primary treatment is surgical resection, but over half of all patients will eventually die of metastatic disease. ${ }^{3}$ Although the rate of progression of advanced colorectal cancer is variable, patients have a median survival of only 6-9 months from the diagnosis of metastatic disease, during which time they may develop various physical and psychological symptoms that detract from their quality of life and often precipitate admission to hospital. ${ }^{4}$

The aims of chemotherapy in patients with advanced colorectal cancer are to prolong survival, control symptoms, and maintain or improve quality of life. There is no universally accepted standard therapy or route of administration, and the duration of treatment varies widely. ${ }^{5}$ Chemotherapy may be administered either systemically via the oral or intravenous route or by hepatic infusion. Hepatic infusion is used because the liver is often the first site of metastatic disease in patients with colorectal cancer and may be the only site of spread in $30-40 \%$ of patients with advanced disease. ${ }^{6}$

Palliative chemotherapy is now offered to an increasing proportion of patients with advanced colorectal cancer. ${ }^{4}$ We conducted this review to assess the evidence that chemotherapy is beneficial in this disease.

\section{Methods}

\section{Criteria for selecting studies}

We included randomised controlled trials that compared palliative chemotherapy with supportive care alone or no chemotherapy for patients with locally advanced (unresectable) or metastatic colorectal cancer. Patients may have received previous chemotherapy as adjuvant treatment after surgery or for metastatic disease. Palliative chemotherapy included any single drug or combination regimen given by any route or schedule. Supportive care was defined as anything other than chemotherapy and included symptom control by local radiotherapy, palliative surgery, pain relief, blood transfusion, and social or psychological support. We did not exclude studies in which a proportion of the control group subsequently received chemotherapy.

\section{Search strategy}

We searched the following electronic databases for eligible trials up to July 1998: Cochrane Controlled Trials Register, Medline, Embase, CancerLit, CINAHL, Healthstar, Science Citation Index, Edina Biosis, Pascal, and Index to Scientific and Technical Proceedings. Details of ongoing or recently completed trials were sought from several sources including the

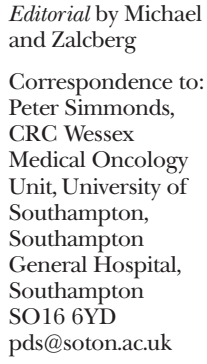

Editorial by Michael and Zalcberg

Correspondence to: Peter Simmonds, CRC Wessex Medical Oncology Unit, University of Southampton, Southampton General Hospital, Southampton SO16 6YD pds@soton.ac.uk

BMJ 2000;321:531-5

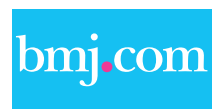

Members of the Colorectal Cancer Collaborative Group and a table giving details of included trials are available on the BMJ's website 
UK Coordinating Committee on Cancer Research trials register, national research register, and physician data query. We also scanned the reference lists of review articles and all primary studies identified, contacted the authors of eligible studies, and hand searched recent conference abstracts to identify further eligible trials.

\section{Review procedures}

All studies were assessed against the inclusion criteria independently by two reviewers, who also extracted data on study methods, participants, interventions, and outcomes from all eligible trials. Any differences were resolved by discussion. Validity was assessed in terms of the method of randomisation, concealment of allocation from trialists, rates of patient withdrawal, and whether the analysis had been performed by intention to treat. ${ }^{7}$ Investigators from all eligible trials were invited to submit individual patient data from their study together with details of trial design and conduct.

The principal outcomes defined in the protocol for this review were survival, disease progression, treatment toxicity, quality of life or relief of symptoms, and cost effectiveness.

\section{Statistical methods}

We analysed individual patient data using the Survival Curve and Hazard Ratio Plot (SCHARP) program (developed by the Medical Research Council Clinical Trials Unit and the Instituto Mario Negri, Milan). Survival analyses were stratified by trial, and the log-rank expected number of events and variance were used to calculate the hazard ratios for individual trials and combined across all trials. The absolute effects of treatment at six months and 12 months were read from simple (non-stratified) Kaplan-Meier curves. ${ }^{8}$ A subgroup analysis was performed to determine whether outcomes differed according to age group. We also conducted a meta-analysis combining the individual patient data analyses and published summary statistics (where available) from the other trials using Metaview 4.0 (Update Software, Oxford). A pooled estimate of effects at prespecified time points was produced by the Mantel-Haenszel (fixed effect) method with results presented as relative risks. The individual patient data analysis is likely to provide the most reliable estimate of the effect of chemotherapy, but the addition of summary statistics from the other trials increases the power of the analysis.

Trials were grouped according to whether chemotherapy was administered regionally or systemically. $\chi^{2}$ tests for heterogeneity were used to test for gross statistical heterogeneity over all trials and for interaction between the subsets of regional versus systemic chemotherapy. When overall results were significant the number needed to treat to have one more person alive was calculated by combining the overall relative risk reduction from the combined meta-analysis with an estimate of the risk of death in the control groups of the trials.

Data on chemotherapy toxicity and quality of life were tabulated but were not combined quantitatively because of significant variations in the method and timing of assessments.

\section{Results}

\section{Description of studies}

Thirteen trials published between 1983 and 1998 met the inclusion criteria (see $B M F$ s website for details). Two trials included patients with other digestive tract tumours in addition to colorectal cancer. ${ }^{910}$ One trial specified an upper age limit of 70 years, ${ }^{11}$ seven trials had an upper age limit of 75 years, ${ }^{92-17}$ and one trial was performed exclusively in patients aged 70 years or older. $^{10}$

Studies of hepatic infusional chemotherapy included only patients with unresectable liver metastases whose primary tumour had been resected, whereas studies of systemic chemotherapy also included patients with locally unresectable disease and extrahepatic metastases. One trial was conducted exclusively in patients who were asymptomatic at trial entry, ${ }^{15}$ whereas all other trials seem to have included both symptomatic and asymptomatic patients, although this is often not stated explicitly.

Seven trials assessed the value of intravenous chemotherapy, ${ }^{9} 10^{14} 1517-19$ one trial evaluated oral chemotherapy, ${ }^{20}$ and five trials evaluated regional chemotherapy delivered via the portal vein or hepatic artery with or without some attempt to disrupt hepatic blood flow. ${ }^{11-131621}$ No two studies used the same treatment combination or schedule, although nine trials used a 5-fluorouracil based chemotherapy regimen. ${ }^{9-15} 1819$ Chemotherapy was given either for a set period (usually six months) or continuously until disease progression. Supportive care interventions were rarely described in detail but included localised radiotherapy, analgesia, corticosteroids, antibiotics, blood transfusion, nutritional support, psychological support, and other symptomatic treatment. In some trials cytotoxic drugs could be given to patients in the supportive care arm under certain conditions (for example, if supportive care did not achieve palliation or once the patient had developed symptoms $)^{9}{ }^{15}$ or at the discretion of the treating clinician. ${ }^{16}{ }^{17} 21$

We were able to confirm that allocation to treatment group was truly random and concealment was adequate in 10 of the trials. ${ }^{911-172021}$ In the remaining three the allocation method was not described, and we were unable to obtain this information from the investigators. ${ }^{10}{ }^{18}{ }^{19}$ In four trials the published analyses were not conducted by intention to treat, ${ }^{11} 121418$ with about $10 \%$ of patients being excluded after randomisation. Individual patient data were obtained from seven studies. ${ }^{9} 121315-1721$ These trials represent 866 of 1365 randomised patients $(63 \%)$.

\section{Survival}

The results of the meta-analysis of individual patient data show that patients in the treatment group had a significantly reduced risk of death (hazard ratio 0.65 ( $95 \%$ confidence interval 0.56 to 0.76 ); fig 1 ). The absolute difference in survival was $16 \%$ at six months $(79 \%$ $v 63 \%)$ and also at 12 months (50\% v 34\%). There was no evidence of gross statistical heterogeneity within subsets of regional and systemic chemotherapy or across all trials $\left(\chi^{2}=5.233, \mathrm{df}=6 ; \mathrm{P}=0.514\right)$. Median survival was estimated to be 8.0 months in the control group and 11.7 months in the chemotherapy group (fig 2). 


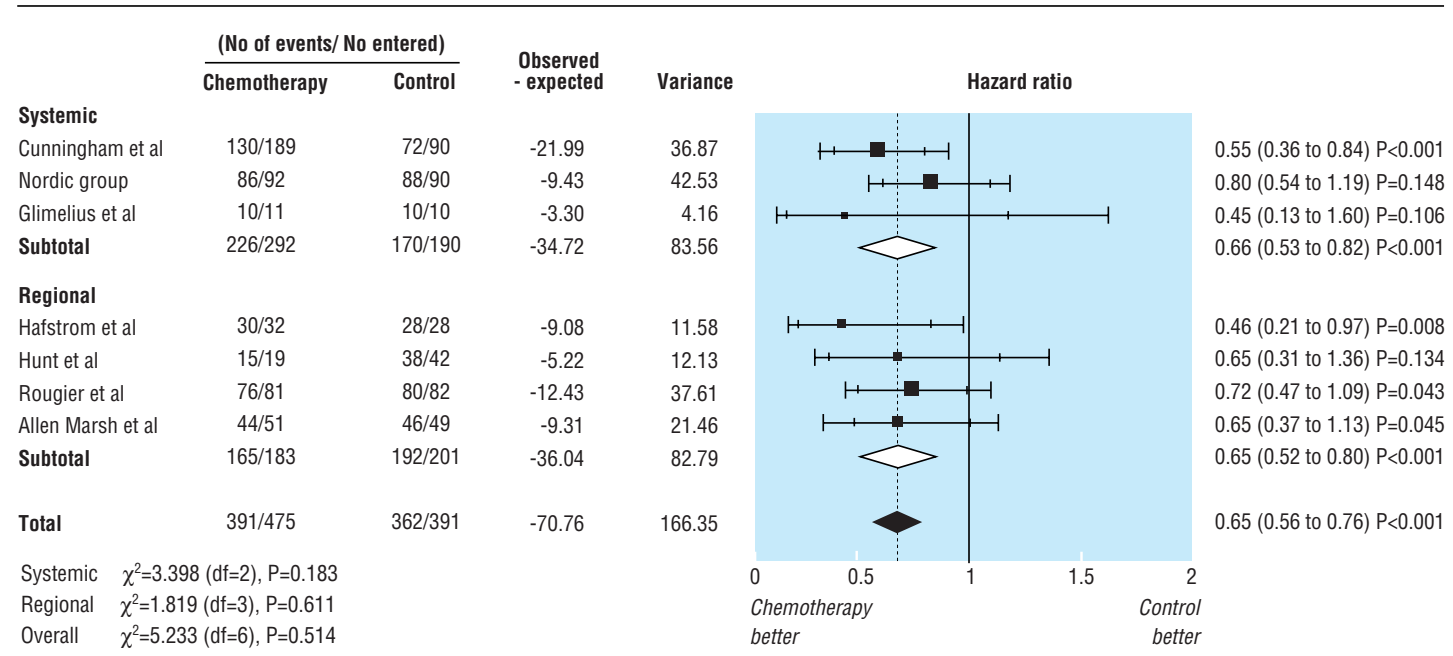

Fig 1 Pooled analysis of individual patient data for survival; data grouped by systemic and regional administration of chemotherapy

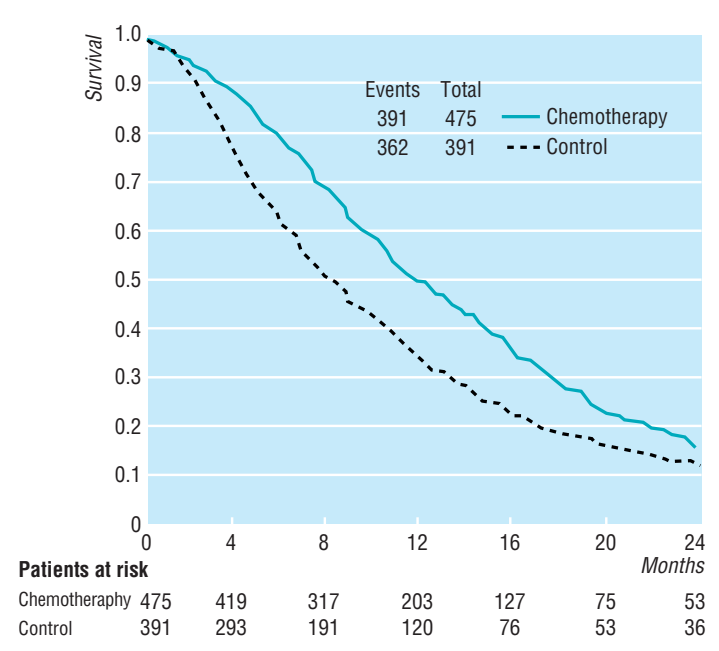

Fig 2 Unstratified Kaplan-Meier plot of overall survival derived from individual patient data from seven randomised controlled trials

Cunningham et al's study ${ }^{17}$ was considered separately in a sensitivity analysis of survival results. This study differed from the others as it evaluated a second line chemotherapy regimen. As it was also the largest study, any differences may have unduly influenced our results. Survival differences between chemotherapy and control groups remained significant even when the results from this study were excluded (hazard ratio 0.69 (0.58 to 0.82$)$ ).

Similar results were obtained in the combined analysis when the published data from the remaining trials were included. ${ }^{11}{ }^{14}{ }^{18-20}$ However, significant statistical heterogeneity, primarily within the systemic chemotherapy trials, was apparent in this analysis $\left(\chi^{2}=\right.$ 28.90, $\mathrm{df}=11 ; \mathrm{P}<0.0001$ at six months). Treatment with palliative chemotherapy was associated with a significantly reduced risk of death at six months (relative risk 0.67 (0.56 to 0.79$)), 12$ months $(0.80(0.73$ to 0.88$))$, 18 months $(0.88$ (0.82 to 0.95$))$, and 24 months (0.92 (0.88 to 0.97$))$ after randomisation. These figures equate to absolute risk reductions of $12.5 \%$ (95\% confidence interval $7.7 \%$ to $17.5 \%), 12.9 \%$ ( $7.6 \%$ to $18.1 \%$ ),
$9.2 \%(4.2 \%$ to $14.1 \%)$, and $6.4 \%(2.1 \%$ to $10.7 \%)$ respectively. From these data, the number needed to treat in order to prevent one additional death at both six and 12 months is 8 (95\% confidence interval 7 to 9 at six months and 6 to 9 at 12 months).

\section{Disease progression}

Only three trials contributed individual patient data on tumour progression (482 patients and 324 cases of tumour progression). ${ }^{9}{ }^{15} 17$ Patients in the treatment group had a significantly reduced risk of progression (hazard ratio $0.51 \quad(0.40$ to 0.64$)$ ). The absolute difference in progression was $25 \%(61 \% v 36 \%)$ at six months and also at 12 months (41\% v 16\%). We found no evidence of statistical heterogeneity across the trials $\left(\chi^{2}=0.03, \mathrm{df}=2, \mathrm{P}=0.985\right)$. Median progression-free survival was estimated to be 4.0 months in the control group and 10.0 months in the chemotherapy group.

Similar results were also seen in the combined analysis of tumour progression, which included published summary statistics from a further three trials at six months ${ }^{111820}$ and 12 months. ${ }^{11}{ }^{18}{ }^{21}$ Chemotherapy treatment was associated with a reduced risk of tumour progression at six months (relative risk 0.68 (0.58 to 0.80$)$ ) and 12 months (relative risk 0.81 ( 0.73 to $0.89))$.

\section{Age related differences in treatment effects}

Very few patients aged 75 years or older were included in the trials from which we obtained individual patient data $(2.5 \%)$. In order to give the analysis greatest power we examined three groups of roughly equal size $(<50$ years, 50-64 years, and $\geqslant 65$ years). We found no relation between age and the effect of treatment on survival $\left(\chi^{2}\right.$ for interaction $=5.039, \mathrm{df}=2, \mathrm{P}=0.08 ; \chi^{2}$ for trend $=0.905, \mathrm{P}=0.341$ (fig 3)). Similarly, no association between age and strength of treatment effect was seen in the analysis of time to progression.

\section{Treatment toxicity}

Data on chemotherapy toxicity were published for 10 out of 13 trials. Only four graded toxicity according to a standardised validated scale (such as the National Cancer Institute common toxicity criteria), and only one study presented the event rates for both the treatment and control arms. ${ }^{17}$ In this study, significantly more 


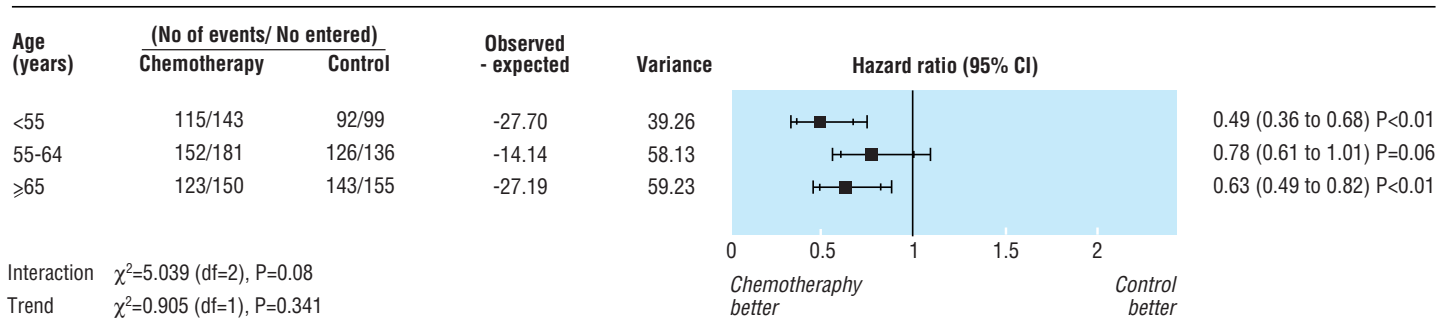

Fig 3 Subgroup analysis of overall survival by age group, derived from individual patient data from seven randomised controlled trials

patients in the treatment group experienced severe adverse events $(79 \% v 67 \%$ grade 3 or 4 on National Cancer Institute common toxicity criteria). Information was not available on the distribution of treatment toxicity according to age group or on whether doses were routinely reduced for elderly patients within these trials. Variations in drugs and lack of objective standardised data meant we were unable to produce a meaningful summary of treatment related toxicities.

\section{Quality of life}

Many of the trials in this analysis were done before validated quality of life instruments specific to patients with cancer were available. Six trials provided information about quality of life. ${ }^{914} 16172022$ All used different assessment instruments, many of which were not cancer specific or validated for patients with advanced cancer. Three trials found superior results in the chemotherapy arm, ${ }^{9}{ }^{17} 22$ two trials found no significant differences between treatment and control groups,${ }^{14}{ }^{16}$ and one trial, in which chemotherapy was ineffective, found quality of life was worse in the treatment group. ${ }^{20}$ Many studies did not provide information about who completed the quality of life instrument and how much help was given by carers and healthcare professionals. It is unclear whether quality of life assessments continued after stopping treatment in many studies.

\section{Cost effectiveness}

The available data were not of sufficient quantity or quality to enable us to assess the economic impact of palliative chemotherapy by secondary economic modelling.

\section{Discussion}

Although colorectal cancer is a major cause of morbidity and mortality, only 1365 patients have been entered into randomised trials comparing chemotherapy with supportive care. The trials are heterogeneous in terms of the patient population, interventions, and control group considered. The pooled results of these trials therefore represent a generalised estimate of the effectiveness of chemotherapy. In addition, a proportion of patients in the control arm of some trials received chemotherapy, so these trials may underestimate differences in survival, disease progression, toxicity, and quality of life.

The results of our meta-analyses using individual patient data alone and in combination with published summary statistics from other trials are broadly similar. They show that for patients with advanced colorectal cancer, treatment with chemotherapy modestly prolongs both the time to tumour progression and survival. Although the benefits of chemotherapy might be expected to be reduced in patients who have had previous chemotherapy, the results of the largest single trial, ${ }^{17}$ in which all patients had received previous chemotherapy, are consistent with the overall outcome of the meta-analysis. Most studies did not differentiate between patients with symptomatic and asymptomatic disease at trial entry, and it is therefore not possible to estimate the benefits for each of these subgroups.

The benefits of chemotherapy must be weighed against treatment toxicity and effect on quality of life, and these outcomes have been inadequately addressed in most trials. Assessment of treatment related toxicity is fundamental in determining the acceptability of chemotherapy as a palliative treatment, yet many trials reported little or no toxicity data and only four used validated assessment scales to classify the severity of toxicities. Only one trial compared toxicity data in the treatment and control groups. ${ }^{17}$ This comparison is important as some symptoms may be related to the underlying disease process rather than to chemotherapy. Maintenance or improvement in quality of life is one of the most important goals of palliative chemotherapy treatment. Despite this, seven of the thirteen trials did not report any assessment of quality of life. Even when quality of life measures were included, the variety of assessment instruments used over varying periods means that it is likely that different aspects of quality of life were assessed in each study, and this makes any direct comparison impossible. Allocation of treatment was not masked from participants, and therefore expectation of benefit or perceptions of suboptimal treatment may have affected quality of life assessments.

Colorectal cancer predominantly affects elderly people, and over half of all deaths occur in people older than $75 .^{23}$ However, elderly patients were under represented in these trials as most imposed an upper age limit for recruitment of subjects. ${ }^{911-17}$ Chemotherapy seemed to be equally effective in the oldest patient group as in younger patients, but the elderly patients included are likely to be highly selected and thus not characteristic of patients in this age group. One trial was conducted solely in patients aged 70 years or over, ${ }^{10}$ but only half of the patients in this study had colorectal cancer and it is not possible to determine the outcome of this subgroup of patients from the published abstract.

Although chemotherapy can prolong life in patients with advanced colorectal cancer, the median survival remains short. Further research is needed to clarify the palliative benefit of chemotherapy. This research should use standardised, validated instruments to examine toxicity, symptom control, and quality of life both 


\section{What is already known on this topic}

Randomised trials comparing palliative chemotherapy with supportive care in patients with advanced colorectal cancer have mostly been underpowered or have not assessed all important outcomes of treatment

Many different treatment regimens are used, the benefits of which are unclear

\section{What this study adds}

Meta-analysis shows that chemotherapy prolongs the time to disease progression and overall survival in patients with advanced colorectal cancer

Data on the effect of chemotherapy on quality of life are inadequate to draw firm conclusions about the palliative benefit of chemotherapy

during and after the completion of treatment. Future trials should not impose upper age limits, and stratification of patients by age at trial entry should be considered to determine whether treatment outcomes vary in different age groups.

We thank the Meta-analysis Group in Cancer (c/o Henri Mondor Hospital, Creteil, France) for providing data for one of the trials, Phil Alderson (UK Cochrane Centre) for guidance when writing the protocol, Jayne Tierney and Lesley Stewart (meta-analysis group, MRC Clinical Trials Unit) for help with the individual patient data meta-analysis, Jon Deeks (Centre for Statistics in Medicine, Oxford) for statistical advice, Andrew Lobb (University of Southampton) for reviewing the search strategy, Jo-anne Gregory (University of Southampton) for administrative support, and Colon Cancer Concern for providing a consumer perspective. The views and opinions expressed are those of the authors and do not necessarily reflect those of the NHS Executive.

Contributors: All members of the group were involved in the protocol design, data extraction, and interpretation of the results and commented on early and final drafts of the paper. PS and Lesley Best performed all searches, determined inclusion, analysed the data, and wrote the paper. Tom Jefferson (Cochrane Economic Analyses Methods Group) provided an economic commentary. PS is the guarantor.

Funding: NHS National Cancer Research and Development Programme.

Competing interests: None declared.

1 Office for National Statistics. Cancer statistics registrations: England and Wales, 1992. London: Stationery Office, 1998.

Office for National Statistics. Mortality statistics: cause, England and Wales 1997. London: Stationery Office, 1997.
3 Cunningham D, Findlay M. The chemotherapy of colon cancer can no longer be ignored. Eur J Cancer 1993;29A:2077-9.

4 Seymour MT, Stenning SP, Cassidy J. Attitudes and practice in the management of metastatic colorectal cancer in Britain. Colorectal Cancer Working Party of the UK Medical Research Council. Clin Oncol 1997;9:248-51

5 Wils J, Sahmoud T, Sobrero A, Bleiberg H, Ahmedzai S, Blazeby J, et al. Evaluation of clinical efficacy of new medical treatments in advanced colorectal cancer. Results of a workshop organized by the EORTC GITCCG. Tumori 1998;84:335-47.

6 tk;4Weiss L, Grundmann E, Torhorst J, Hartveit F, Moberg I, Eder M. Hematogenous metastatic patterns in colonic carcinoma: an analysis of 1541 necropsies. J Pathol 1986;150:195-203.

7 Jadad AR, Moore RA, Carroll D, Jenkinson C, Reynolds DJ, Gavaghan DJ et al. Assessing the quality of reports of randomized clinical trials: is blinding necessary? Control Clin Trials 1996;17:1-12.

8 Kaplan EL, Meier P. Nonparametric estimation from incomplete observations. J Am Statist Ass 1958;53:479-86.

9 Glimelius B, Hoffman K, Graf W, Haglund U, Nyren O, Pahlman L, et al. Cost-effectiveness of palliative chemotherapy in advanced gastrointestinal cancer. Ann Oncol 1995:6:267-74.

10 Beretta G, Bollina R, Cozzi C, Morabito A. Should we consider the weekly chemotherapy with fluorouracil + racemic folic acid a standard treatment for advanced/metastatic carcinoma of the digestive tract in elderly patients? Proc Am Soc Clin Oncol 1997;16:A920.

11 Gerard A, Buyse M, Pector JC, Bleiberg H, Arnaud JP, Willems G, et al. Hepatic artery ligation with and without portal infusion of 5-FU. A randomized study in patients with unresectable liver metastases from colorectal carcinoma. The EORTC Gastrointestinal Cancer Cooperative Group (GI group). Eur J Surg Oncol 1991;17:289-94.

12 Hafstrom L, Engaras B, Holmberg SB, Gustavsson B, Jonsson P, Linder P, et al. Treatment of liver metastases from colorectal cancer with hepatic artery occlusion, intraportal 5-fluouracil infusion, and oral allopurinol. Cancer 1994;74:2749-56.

13 Hunt TM, Flowerdew AD, Birch SJ, Williams JD, Mullee MA, Taylor I. Prospective randomized controlled trial of hepatic arterial embolization or infusion chemotherapy with 5 -fluorouracil and degradable starch microspheres for colorectal liver metastases. Br J Surg 1990;77:779-82.

14 Scheithauer W, Rosen H, Kornek GV, Sebesta C, Depisch D. Randomised comparison of combination chemotherapy plus supportive care with supportive care alone in patients with metastatic colorectal cancer. $B M J$ 1993:306:752-5.

15 Nordic Gastrointestinal Tumor Adjuvant Therapy Group. Expectancy or primary chemotherapy in patients with advanced asymptomatic colorectal cancer: a randomized trial. J Clin Oncol 1992;10:904-11.

16 Allen Mersh TG, Earlam S, Fordy C, Abrams K, Houghton J. Quality of life and survival with continuous hepatic-artery floxuridine infusion for colorectal liver metastases. Lancet 1994:344:1255-60.

17 Cunningham D, Pyrhonen S, James RD, Punt CJA, Hickish TF, Heikkila R, et al. Randomised trial of irinotecan plus supportive care versus supportive care alone after fluorouracil failure for patients with metastatic colorectal cancer. Lancet 1998;352:1413-8.

18 Yorkshire Gastrointestinal Tumour Group. Chemotherapy after palliative resection of colorectal cancer. Br J Surg 1984;71:283-6.

19 Chisholm E, Malhotra A, Giles GR. Quadruple chemotherapy for advanced colorectal cancer. Clin Oncol 1983;9:185-6.

20 Smyth JF, Hardcastle JD, Denton G, Alderson D, Grace RH, Mansi JL, et al. Two phase III trials of tauromustine (TCNU) in advanced colorectal cancer. Ann Oncol 1995;6:948-9.

21 Rougier P, Laplanche A, Huguier M, Hay JM, Ollivier JM, Escat J, et al Hepatic arterial infusion of floxuridine in patients with liver metastases from colorectal carcinoma: long-term results of a prospective randomized trial. J Clin Oncol 1992;10:1112-8.

22 Glimelius B, Graf W, Hoffman K, Pahlman L, Sjoden PO, Wennberg A. General condition of asymptomatic patients with advanced colorectal cancer receiving palliative chemotherapy. A longitudinal study. Acta Oncol 1992;31:645-51.

23 Silverberg E, Boring CC, Squires TS. Cancer statistics, 1990. CA Cancer J Clin 1990;40:9-26.

(Accepted 17 April 2000)

\section{Email submissions from outside the United Kingdom}

We are now offering an email submission service for authors from outside the UK. The address is papers@bmj.com Ideally our email server would link seamlessly with our manuscript tracking system, but for now it does not, which is why we are offering the service only to authors outside the UK. Most post in the UK arrives the next day, so UK authors have the least to gain in speed of delivery from email delivery. As soon as our systems improve we will invite email submissions from everyone.

If you choose to send your submission by email please would you send the text and any tables and figures as attached files, together with a covering letter giving all your contact details (postal address, phone, fax, and email address). We can read files created with most word processing, graphics, and spreadsheet programs. When your submission is received in our email box you will receive an automatic acknowledgment to show that it has arrived. If the submission is incomplete we will contact you and ask you to resend the missing information.

Once the submission is complete we will register it on our manuscript tracking system and you will receive a standard acknowledgment in the post.

Letters to the editor should continue to be sent direct to bmj.com as rapid responses or toletters@bmj.com 